\title{
Slopes of trigonal fibred surfaces and of higher dimensional fibrations
}

\author{
Miguel Ángel BARJA And Lidia STOPPINO
}

\begin{abstract}
We give lower bounds for the slope of higher dimensional fibrations $f: X \longrightarrow B$ over curves under conditions of GIT-semistability of the fibres, using a generalization of a method of Cornalba and Harris. With the same method we establish a sharp lower bound for the slope of trigonal fibrations of even genus and general Maroni invariant; this result in particular proves a conjecture due to Harris and Stankova-Frenkel.
\end{abstract}

Mathematics Subject Classification (2000): 14J10 (primary); 14J29, 14D06 (secondary).

\section{Introduction and preliminaries}

Given a fibration over a curve $f: X \longrightarrow B(X, B$ complex, projective varieties, $B$ a smooth curve, $f$ surjective with connected fibres) and a line bundle $\mathcal{L}=\mathcal{O}_{X}(L)$ on $X$, we can define the slope of the pair $(f, \mathcal{L})$ to be the quotient

$$
s(f, \mathcal{L})=\frac{L^{n}}{\operatorname{deg} f_{*} \mathcal{L}}
$$

provided $\operatorname{deg} f_{*} \mathcal{L} \neq 0$, where $n$ is the dimension of $X$. When $\mathcal{L}=\omega_{f}$, the relative dualizing sheaf of $f$, we simply call it the slope of $f$ and will denote it as $s(f)$. Lower bounds for the slope have been extensively studied in the literature (e.g., $[1,4,5,15,16,26,32])$ for the case of fibred surfaces $(n=2)$ and some results are known in dimension $n=3[3,22]$.

In this paper, we study this problem using a generalized version of a theorem of Cornalba-Harris $[7,26]$. This method provides a general result to produce lower bounds of $s(f, \mathcal{L})$ provided the pair $\left(F,\left|\mathcal{L}_{\mid F}\right|\right)$ (where $F$ is a general fibre of $f$ ) is

The first author was partially supported by MEC-MTM2006-14234-C02-02 and by Generalitat de Catalunya 2005SGR00557. The second one was partially supported by PRIN 2005 "Spazi di Moduli e Teorie di Lie", FAR 2008 (Pavia) Varietà algebriche, calcolo algebrico, grafi orientati e topologici.

Received September 10, 2008; accepted in revised form February 5, 2009. 
semistable in the sense of Geometric Invariant Theory. In Section 2 we recall this result and derive the following consequences (see Corollaries 2.2 and 2.3 for more detailed statements).

Theorem 1.1. Let $X$ be a variety of dimension $n$ and $\phi: X \longrightarrow B$ a flat morphism over a curve. Let $F$ be a general fibre of $f$. Suppose that $\left(F,\left|\mathcal{L}_{|F|}\right|\right)$ is Hilbert semistable. Assume moreover that $R^{i} f_{*} \mathcal{L}^{h}=0$ for $i>0$ and $h \gg 0$. Then

i) If $\left|\mathcal{L}_{\mid F}\right|$ induces an embedding, we have

$$
L^{n} \geq n \frac{\left(L_{\mid F}\right)^{n-1}}{h^{0}\left(F, \mathcal{L}_{\mid F}\right)} \operatorname{deg} f_{*} \mathcal{L} .
$$

ii) If $\left|\mathcal{L}_{\mid F}\right|$ induces an generically finite rational map onto a variety of degree $d$ and $f_{*} \mathcal{L}^{h}$ is nef, we have

$$
L^{n} \geq n \frac{d}{h^{0}\left(F, \mathcal{L}_{\mid F}\right)} \operatorname{deg} f_{*} \mathcal{L}
$$

The method applied directly to families of canonical varieties would give very interesting higher dimensional slope inequalities. However, already in the case of surfaces it is very hard to check the stability assumption. For instance it is not known if a "general" surface of general type satisfies it or not. The Hilbert stability of hypersurfaces with log-canonical singularities can be derived by a result of Kim and Lee [14] (see also [17]), which relates the stability of a hypersurface $X \subset \mathbb{P}^{n}$ to the log-canonical threshold of the couple $\left(\mathbb{P}^{n}, X\right)$. The case of log-terminal singularities was established by Tian [30] using methods of differential geometry. Then we can deduce a bound on the slope, when the fibres are hypersurfaces which are canonical, i.e., such that their canonical map is birational (see 2.5).

Theorem 1.2. Let $f: X \longrightarrow B$ be a surjective flat morphism from a $\mathbb{Q}$-factorial projective $n$-fold $X$ to a smooth complete curve $B$. Suppose that the fibration is relatively minimal and that the general fibres $F$ are minimal canonical varieties with $p_{g}=n+1, K_{F}^{n-1}=n+2$, such that its canonical image has at most logcanonical singularities. Then

$$
K_{f}^{n} \geq \frac{n(n+2)}{(n+1)} \operatorname{deg} f_{*} \omega_{f} .
$$

Examples of such fibres $F$ are smooth hypersurfaces of $\mathbb{P}^{n}$ of degree $n+2$.

It is worth mentioning that this theorem is the first result proving lower bounds for the slope of fibrations of dimension higher than 3 .

Eventually we give a new evidence of the necessity of the stability assumption in the $\mathrm{C}-\mathrm{H}$ theorem (Remark 2.6).

Section 3 is devoted to the study of a particular type of fibred surfaces, the so called trigonal fibrations (i.e., when the general fibre is a trigonal curve). An 
intensively studied problem in the last decades is to find of lower bounds for the slope of fibred surfaces. In general, the so called slope inequality holds [7, 26, 32]

$$
s(f) \geq 4-\frac{4}{g} .
$$

It is sharp and equality is satisfied only for certain kind of hyperelliptic fibrations $[1,26]$.

There are several reasons to conjecture that the gonality of the general fibre of $f$ has an increasing influence on the lower bound of the slope (see $[4,15,25]$ and Remark 3.6). So the next natural problem in this framework is the one of studying trigonal fibrations.

The main known results are the following:

(Konno, [16]) If $f: X \longrightarrow B$ is a trigonal fibration of genus $g \geq 6$, then

$$
s(f) \geq \frac{14(g-1)}{3 g+1} .
$$

(Stankova-Frenkel, [25, Proposition 9.2 and Proposition 12.3]) If $f: X \longrightarrow B$ is a trigonal semistable fibration, then

$$
s(f) \geq \frac{24(g-1)}{5 g+1} .
$$

This bound is sharp, and if equality holds the general fibres have Maroni invariant $\geq 2$.

Moreover, if $g$ is even and the following conditions hold:

- the general fibres have Maroni invariant 0;

- the singular fibres are irreducible and have only certain kind of singularities; then the slope satisfies the bound

$$
s(f) \geq \frac{5 g-6}{g} .
$$

Harris and Stankova-Frenkel conjecture [25, Conjecture 12.1] the bound (1.3) to hold without the extra condition on singular fibres.

It has to be remarked that the bounds (1.2) and (1.3), although better than (1.1), are proved to hold only for semistable fibrations i.e. for fibred surfaces such that all the fibres are semistable curves in the sense of Deligne-Mumford; this is, from the point of view of fibred surfaces, a strong restriction. Indeed, starting from any fibred surface, one can construct a semistable one by the process of semistable reduction, but the slope cannot be controlled through this process, as shown in [27].

The main result of Section 3 is the following (Theorem 3.3): 
Theorem 1.3. Let $f: S \longrightarrow B$ be a relatively minimal fibred surface such that the general fibre $C$ is a trigonal curve of even genus $g \geq 6$ with Maroni invariant 0. Then the slope satisfies inequality (1.3).

Observe that we are not assuming $f$ to be semistable. In particular, we give a positive answer to the Harris-Stankova-Frenkel conjecture.

Moreover, in Theorem 3.3, we prove at the same time that (1.3) holds for any fibration of genus 6 whose general fibres have a $g_{5}^{2}$, thus improving the bound proved by Konno in [16], which is $96 / 25$.

This result can be seen as a first step when searching for an increasing dependence of the slope from the gonality of the general fibres. The assumption on the Maroni invariant assures that the fibres are general in the locus of trigonal curves, consistently with the conjectures (see Remark 3.6).

We prove this theorem applying the $\mathrm{C}-\mathrm{H}$ method to a fibred 3-fold naturally associated to the fibred surface; indeed the slope of $f$ is related to the one of the relative quadric-hull $W \longrightarrow B$ of the trigonal fibration $f: S \longrightarrow B(c f .[6,16])$, for a suitable line bundle on it. In the case of Maroni invariant 0 , the general fibre of the hull is $\mathbb{P}^{1} \times \mathbb{P}^{1}$, embedded as a surface of minimal degree in $\mathbb{P}^{g-1}$, this embedding being GIT semistable by a result of Kempf [13].

ACKnowledgements. We wish to thank Maurizio Cornalba, Andreas Leopold Knutsen and Andrea Bruno for many helpful conversations on this topic.

\section{The Cornalba-Harris Method and the slope of fibrations}

We work over the complex field $\mathbb{C}$. Let $X$ be a variety (an integral separated scheme of finite type over $\mathbb{C}$ ), with a linear system $V \subseteq H^{0}(X, \mathcal{L})$, for some line bundle $\mathcal{L}$ on $X$. Fix $h \geq 1$ and call $G_{h}$ the image of the natural homomorphism

$$
H^{0}\left(\mathbb{P}^{s}, \mathcal{O}_{\mathbb{P}}(h)\right)=\operatorname{Sym}^{h} V \stackrel{\varphi_{h}}{\longrightarrow} H^{0}\left(X, \mathcal{L}^{h}\right) .
$$

Set $N_{h}=\operatorname{dim} G_{h}$ and take exterior powers $\wedge^{N_{h}} \operatorname{Sym}^{h} V \stackrel{\wedge^{N_{h}} \varphi_{h}}{\longrightarrow} \wedge^{N_{h}} G_{h}=\operatorname{det} G_{h}$. We can see $\wedge^{N_{h}} \varphi_{h}$ as a well-defined element of $\mathbb{P}\left(\wedge^{N_{h}} \operatorname{Sym}^{h} V^{\vee}\right)$.

With the above notations, we call $\wedge^{N_{h}} \varphi_{h} \in \mathbb{P}\left(\wedge^{N_{h}} \operatorname{Sym}^{h} V^{\vee}\right)$, the generalized $h$-th Hilbert point associated to the couple $(X, V)$. If $V$ induces an embedding, then for $h \gg 0$ the homomorphism $\varphi_{h}$ is surjective and it is the classical $h$-th Hilbert point.

Fix a basis and consider the obvious representation $S L(s+1, \mathbb{C}) \longrightarrow S L(V)$; we get an induced natural action of $S L(s+1, \mathbb{C})$ on $\mathbb{P}\left(\wedge^{N} \operatorname{Sym}^{h} V^{\vee}\right)$, and we can introduce the associated notion of GIT (semi)stability: we say that the $h$-th generalised Hilbert point of the couple $(X, V)$ is semistable (respectively stable) if it is GIT semistable (respectively stable) with respect to the natural $S L(s+1, \mathbb{C})$-action. 
We say that $(X, V)$ is generalised Hilbert stable (respectively semistable) if its generalised $h$-th Hilbert point is stable (respectively semistable) for infinitely many integers $h>0$.

We state a generalised version of the Cornalba-Harris theorem.

Theorem 2.1. [26, Theorem 1.5] Let $X$ be a variety of dimension $n$ and $\phi: X \longrightarrow$ $B$ a flat morphism over a curve, and call $F$ a general fibre. Let $\mathcal{L}$ be a line bundle on $X$. Let $h$ be a positive integer, and assume that $\left(F,\left|\mathcal{L}_{|F|}\right|\right)$ has semistable generalised h-th Hilbert point. Consider a vector subbundle $\mathcal{G}_{h}$ of $\phi_{*} \mathcal{L}^{h}$ such that $\mathcal{G}_{h}$ contains the image of the morphism of sheaves

$$
\operatorname{Sym}^{h} \phi_{*} \mathcal{L} \longrightarrow \phi_{*} \mathcal{L}^{h}
$$

and coincides with it at general $t \in B$.

Then the line bundle

$$
\mathcal{F}_{h}:=\left(\operatorname{det} \phi_{*} \mathcal{L}\right)^{-h N_{h}} \otimes\left(\operatorname{det} \mathcal{G}_{h}\right)^{r},
$$

where $r:=h^{0}\left(F, \mathcal{L}_{\mid F}\right)$, and $N_{h}:=\operatorname{rank} \mathcal{G}_{h}$, is effective.

Corollary 2.2. Let $X$ be a variety of pure dimension $n$ and $\phi: X \longrightarrow B$ a flat proper morphism over a curve, and call $F$ a general fibre. Let $\mathcal{L}$ be a line bundle on $X$ such that $\left(F,\left|\mathcal{L}_{\mid F}\right|\right)$ is generalized Hilbert semistable. Suppose moreover that

(1) the linear system $\left|\mathcal{L}_{\mid F}\right|$ induces an embedding of the general fibre $F$;

(2) the sheaves $R^{i} \phi_{*} \mathcal{L}^{h}$ vanish for $i>0$ for h large enough. ${ }^{1}$

Then, the following inequality holds

$$
L^{n} \geq n \frac{\left(L_{\mid F}\right)^{n-1}}{h^{0}\left(F, \mathcal{L}_{\mid F}\right)} \operatorname{deg} \phi_{*} \mathcal{L} .
$$

Proof. By the first assumption, we can apply Theorem 2.1 with $\mathcal{G}_{h}=\phi_{*} \mathcal{L}^{h}$. Hence, for infinitely many $h>0$ the line bundle $\mathcal{F}_{h}$ is effective. Now, under our assumptions $\operatorname{deg} \mathcal{F}_{h}$ is a degree $n$ polynomial in $h$ with coefficients in the rational Chow ring $C H^{1}(X)_{\mathbb{Q}}$. Its leading coefficient has to be pseudo-effective, hence to have non-negative degree. The statement now follows from an intersectiontheoretical computation. Indeed, the Riemann-Roch formula for singular varieties (cf. [8, Corollary 18.3.1]) implies the following expansions:

$$
N_{h}=\frac{\left(L_{\mid F}\right)^{n-1}}{(n-1) !} h^{n-1}+O\left(h^{n-2}\right),
$$

and

$$
\operatorname{deg} \phi_{*} \mathcal{L}^{h}=\operatorname{deg} \phi_{!} \mathcal{L}^{h}=\frac{L^{n}}{n !} h^{n}+O\left(h^{n-1}\right),
$$

because the higher direct images vanish by the second assumption.

${ }^{1}$ This happens for instance if $\mathcal{L}$ is $\phi$-ample. 
Corollary 2.3. Let $X$ be a variety of pure dimension $n$ and $\phi: X \longrightarrow B$ a flat proper morphism over a curve, and call $F$ a general fibre. Let $\mathcal{L}$ be a line bundle on $X$ such that $\left(F,\left|\mathcal{L}_{|F|}\right|\right)$ is generalized Hilbert semistable. Suppose moreover that for large enough $h$

(1) the linear system $\left|\mathcal{L}_{\mid F}\right|$ induces a generically finite rational map on the image of $F$;

(2) the vector bundle $\phi_{*} \mathcal{L}^{h}$ is nef (i.e. every quotient has non-negative degree);

(3) the sheaves $R^{i} \phi_{*} \mathcal{L}^{h}$ vanish for $i>0$.

Then the following inequality holds

$$
L^{n} \geq n \frac{d}{h^{0}\left(F, \mathcal{L}_{\mid F}\right)} \operatorname{deg} \phi_{*} \mathcal{L},
$$

where $d$ is the degree of the image $\phi(F) \subseteq \mathbb{P}^{r}$.

Proof. Let $h$ be large enough. By the nef-ness assumption on $\phi_{*} \mathcal{L}^{h}$, the degree of $\mathcal{F}_{h}$ is smaller or equal to the degree of $\left(\operatorname{det} \phi_{*} \mathcal{L}\right)^{-h N_{h}} \otimes\left(\operatorname{det} \phi_{*} \mathcal{L}^{h}\right)^{r}$. Then the statement follows applying Riemann-Roch for singular varieties as in the previous corollary, observing that (by the first assumption) $N_{h}=d h^{n-1} /(n-1) !+$ $O\left(h^{n-2}\right)$.

In particular, using the relative canonical divisor, we can obtain the following result on the slopes of families of certain canonical varieties.

Remark 2.4. Let $\phi: X \longrightarrow B$ be a fibration of a normal $\mathbb{Q}$-factorial variety with at most canonical singularities over a curve. Under these assumptions $K_{X}$ (and $\left.K_{\phi}=K_{X}-\phi^{*} K_{B}\right)$ is a Weil, $\mathbb{Q}$-Cartier divisor. We can consider its associated divisorial sheaves $\omega_{X}$ and $\omega_{\phi}$. Suppose that the canonical sheaf $\omega_{X}$ is $\phi$-nef, and that on a general fibre $F$ the canonical divisor $\omega_{F}=\omega_{\phi \mid F}$ induces a generalized Hilbert semistable map which is finite on the image of $F$. Then the following inequality holds

$$
K_{\phi}^{n} \geq n \frac{d}{p_{g}(F)} \operatorname{deg} \phi_{*} \omega_{\phi},
$$

where $p_{g}(F)=h^{0}\left(F, K_{F}\right)$ and $d$ is the degree of the canonical image of the general fibre $F$ in $\mathbb{P}^{p_{g}(F)-1}$. In particular, if $\omega_{F}$ induces a birational morphism, $d=K_{F}^{n-1}$.

Indeed, we can apply Corollary 2.3 to the relative canonical sheaf: $\mathcal{L}=\omega_{\phi}$. The second assumption is satisfied by [31], while the third one derives from the relative nefness of $\omega_{X}$, using the relative version of Kawamata-Viehweg vanishing Theorem (see for instance [12, Theorem 1.2.3]).

Although it is difficult to check the stability assumption for varieties of dimension bigger than 1, by a result of Kim and Lee [14] we have:

(Kim and Lee) Any normal hypersurface $F \subseteq \mathbb{P}^{n}$ of degree $d \geq n+2$ with only log-canonical singularities is Hilbert stable. 
This result follows easily from the main result in [14], recalling that Chow stability implies generalized Hilbert stability.

One should compare this result with [30], where Hilbert stability is proved under the assumption of log-terminal singularities, using differential geometry techniques.

We will say that a variety is canonical if its canonical map is birational onto its image. Hence we can state:

Theorem 2.5. Let $\phi: X \longrightarrow B$ be a surjective flat morphism from a $\mathbb{Q}$-factorial projective $n$-fold $X$ to a smooth complete curve $B$. Suppose that $K_{\phi}$ is $\phi$-nef and that the general fibres $F$ are minimal canonical varieties (of dimension $n-1$ ) with $p_{g}=n+1, K_{F}^{n-1}=n+2$ whose canonical image has at most log-canonical singularities. Then

$$
K_{\phi}^{n} \geq \frac{n(n+2)}{n+1} \operatorname{deg} \phi_{*} \omega_{\phi}
$$

Proof. Note that the generalized Hilbert stability of the canonical morphism $\varphi_{\left|K_{F}\right|}$ of the general fibre $F$ is equivalent to the Hilbert stability of the embedding of the image $\bar{F}=\varphi_{\left|K_{F}\right|}(F) \hookrightarrow \mathbb{P}^{n}\left(c f\right.$. [26]). Now $\bar{F} \subset \mathbb{P}^{n}$ is Hilbert stable by Kim and Lee's result above. The proof is then straightforward using the argument of Remark 2.4.

For instance a one-parameter family of surfaces with $p_{g}=4 q=0$ and $K^{2}=5$ such that the general fibre is of type $(I)$ in Horikawa classification [11, Theorem 1, Section 1] satisfies the conditions of the above theorem; indeed these surfaces have base-point-free birational canonical map, and their canonical images are quintic surfaces in $\mathbb{P}^{3}$ with at most rational double points.

Remark 2.6. We can now give a new example that show the fact that the stability condition in the $\mathrm{C}-\mathrm{H}$ method is necessary, in addition to the one given by Morrison in [7, Section 3].

In [22], Example on page 664, a fibred 3-fold $\phi: T \longrightarrow B$ is constructed fitting in the following diagram

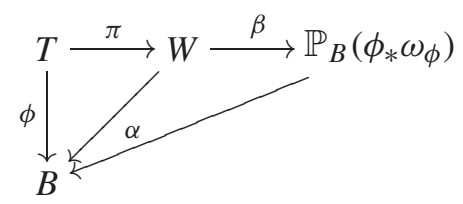

such that

- the general fibre of $\phi$ is a surface of general type with $p_{g}=4, q=0$ and $K_{F}^{2}=4$ whose canonical map is a degree 2 base point free map onto a quadric cone in $\mathbb{P}^{3}$;

- the map $\pi$ is a smooth double cover of a $\mathbb{P}^{2}$-bundle $W$ over $B$ such that $\phi_{*} \omega_{\phi}=$ $\alpha_{*}\left(\omega_{\alpha} \otimes \mathcal{L}\right) \oplus \alpha_{*} \omega_{\alpha}=\alpha_{*}\left(\omega_{\alpha} \otimes \mathcal{L}\right)$ (because $\alpha_{*} \omega_{\alpha}=0$, being the generic fibre of $\alpha$ a rational surface); 
- the composition $\beta \circ \pi$ is the relative canonical map of $\phi$;

- the slope of $\phi$ is 20/7 (see [22, page 665] with $e=2$ ).

On the other hand, as $\omega_{\alpha} \otimes \mathcal{L}$ induces on the general fibres of $\alpha$ the natural map as a quadric cone in $\mathbb{P}^{3}$, by the same argument used by Konno in [16, Lemma 1.1], we can conclude that $R^{i} \alpha_{*}\left(\omega_{\alpha} \otimes \mathcal{L}\right)^{h}=0$ for $i, h>0$.

It is well known that the quadric cone is Hilbert unstable. Assume nevertheless that we could apply Theorem 2.1 to $\omega_{\phi}$. Consider the morphism of sheaves

$$
\operatorname{Sym}^{h} \phi_{*} \omega_{\phi} \longrightarrow \phi_{*} \omega_{\phi}^{h}
$$

We can choose as $\mathcal{G}_{h}$ (in the notations of Theorem 2.1) the sheaf $\alpha_{*}\left(\omega_{\alpha} \otimes \mathcal{L}\right)^{h}$. Computing now the degree 3 coefficient of $\operatorname{deg} \mathcal{F}_{h}$, we obtain

$$
\frac{K_{\phi}^{3}}{2}=\pi^{*}\left(K_{\alpha}+L\right)^{3} \geq \frac{3}{2} \operatorname{deg} \phi_{*} \omega_{\phi}
$$

and hence the slope would be at least 3 , a contradiction.

\section{The slope of trigonal fibrations}

Let $f: S \longrightarrow B$ be a relatively minimal fibred surface such that the general fibre $C$ is a trigonal curve of genus $g$.

Remark 3.1. If $C$ is a trigonal curve of genus $g$, it is a well known fact (see for instance $[18,23,24])$ that its canonical image lives on a Hirzebruch surface $\mathbb{F}_{c}=$ $\mathbb{P}\left(\mathcal{O}_{\mathbb{P}^{1}} \oplus \mathcal{O}_{\mathbb{P}^{1}}(c)\right)$ embedded in $\mathbb{P}^{g-1}$ as a surface of minimal degree. The surface $\mathbb{F}_{c}$ is the intersection of quadrics containing the canonical image of $C$ in $\mathbb{P}^{g-1}$; from a more geometric point of view, it is the rational normal scroll generated by the lines spanned by the divisors in the $g_{3}^{1}$ on the canonical image of $C$. The number $c$ is called the Maroni invariant of $C$; it has the same parity of $g$, and satisfies the inequality $c \leq(g+2) / 3(c f$. [23]).

It has been shown in [19] that the Maroni invariant is an upper semicontinuos function on the trigonal locus $\mathcal{D}_{3}$, and hence a general genus $g$ trigonal curve has Maroni invariant 0 (respectively 1 ) if $g$ is even (respectively odd). The locus of points in $\mathcal{D}_{3}$ corresponding to curves with Maroni invariant $>1$ has codimension 1 the even case, while it has strictly bigger codimension in the odd one.

We can extend the construction mentioned above on the fibres of $f$ to a relative setting, using the so-called relative hyperquadric hull (see e.g. [16] and [6]). Consider the relative canonical image of $S$ :

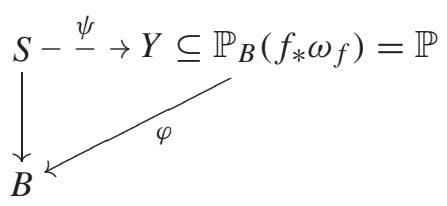


If $\mathcal{A} \in \operatorname{Pic} B$ is ample enough it can be easily checked that we have an epimorphism

$$
H^{0}\left(\mathcal{J}_{Y, \mathbb{P}}(2) \otimes \varphi^{*}(\mathcal{A})\right) \longrightarrow H^{0}\left(\mathcal{J}_{F, \mathbb{P}^{g-1}}(2)\right) .
$$

Let $W_{0}$ be the horizontal irreducible component of the base locus of the linear system on $\mathbb{P}$ given by the sections of $H^{0}\left(\mathcal{J}_{Y, \mathbb{P}}(2) \otimes \varphi^{*}(\mathcal{A})\right)$ ). Since the general fibre $C$ is trigonal, $W_{0}$ is a threefold fibred over $B$ by rational surfaces of minimal degree. Notice moreover that for $g \geq 5$ the singular locus of $W_{0}$ is contained in a finite number of fibres. Let $W$ be a desingularization of $W_{0}$ and let $L$ be the pull-back of the tautological divisor of $\mathbb{P}$ to $W$. We will call $W$ the relative quadric hull associated to $f$ and denote by $\phi: W \longrightarrow B$ the induced fibration. The fibre of $\phi$ over general $t \in B$ coincides with the one of $W_{0}$ (hence it is the rational normal scroll associated to the fibre of $S$ over $t$ ).

The main facts we will need about the divisor $L$ have been proved by Konno in [16] ( $c f$. Lemma 1.1 and Lemma 1.2).

\section{Proposition 3.2 (Konno).}

i) $\phi_{*} \mathcal{O}_{W}(L)=f_{*} \omega_{f}$;

ii) $R^{p} \phi_{*} \mathcal{O}_{W}(h L)=0$ for $p, h>0$;

iii) $K_{f}^{2} \geq 2 \operatorname{deg} f_{*} \omega_{f}+L^{3}$.

We can now state the main result of this section.

Theorem 3.3. Let $f: S \longrightarrow B$ be a relatively minimal fibred surface such that the general fibre $C$ is either:

- a trigonal curve of even genus $g \geq 6$ and zero Maroni invariant;

- a curve of genus 6 with a $g_{5}^{2}$.

Then

$$
s_{f} \geq \frac{5 g-6}{g},
$$

and this bound is sharp.

Proof. Using the relative quadric hull associated to $f$, the general fibre $F$ of $\phi: W \longrightarrow B$ is just $\mathbb{P}^{1} \times \mathbb{P}^{1}$ in the trigonal case and $\mathbb{P}^{2}$ in the case of a plane quintic. The restriction of $L$ to $F$ induces a complete embedding of $F$ in $\mathbb{P}^{g-1}$ as a surface of minimal degree. This is Hilbert semistable according, for instance, to a result of Kempf ( $c f$. [13, Corollary 5.3]). Moreover, $\mathcal{O}_{W}(L)$ has no higher relative cohomology by Proposition 3.2, (ii). We can therefore apply Corollary 2.2 and conclude that

$$
L^{3} \geq 3 \frac{g-2}{g} \operatorname{deg} \phi_{*} \mathcal{O}_{W}(L) .
$$

The statement now follows using inequality (i) and (iii) of Proposition 3.2.

As for the sharpness of this bound, we refer to Example 3.4 below. 
Example 3.4. Using a construction of Tan [29], we can prove that any trigonal curve with Maroni invariant strictly smaller than $(g+2) / 9$ can be realized as the fibre of a semistable fibration over $\mathbb{P}^{1}$ with slope $(5 g-6) / g$. Let $C$ be a trigonal curve with Maroni invariant $c$. Recall that Pic $\mathbb{F}_{c}=\mathbb{Z}[\ell] \oplus \mathbb{Z}[f]$, where $\ell$ is the negative section $\left(\ell^{2}=-c\right)$ and $f$ is a fibre of the ruling $\mathbb{F}_{c} \longrightarrow \mathbb{P}^{1}$. The class of $C \subset \mathbb{F}_{c}$ is $3 \ell+k f$, where $k=(g+2+3 c) / 2$.

As proved for instance in [10, V. Corollary 2.18], the linear system $|3 \ell+k f|$ is very ample if and only if $k>6 c$, that is $9 c<g+2$. In this case, we can choose a general pencil in $|C|$. It has $C^{2}=(3 \ell+k f)^{2}=6 k-9 c=3 g+6$ base points. Let $S$ be the blow up of $\mathbb{F}_{c}$ in these base points, and $f: S \longrightarrow \mathbb{P}^{1}$ the induced fibration. Computing the relative invariants, we obtain $K_{f}^{2}=5 g-6$ and $\chi_{f}=g$.

In [15, Example 4.6], other examples reaching the bound are provided, satisfying the condition that the bundle $f_{*} \omega_{f}$ is semistable.

Remark 3.5. The higher Maroni invariant cases cannot be treated with the $\mathrm{C}-\mathrm{H}$ method. Recall that the general fibre of $W$ is an Hirzebruch surface $\mathbb{F}_{c}$ embedded in $\mathbb{P}^{g-1}$ by the divisor $D=\ell+\frac{g+c-2}{2} f ; D$ is a "good" divisor in the notation of [20], and by Theorem 6.5 of the same paper, the associated embedding is Chow unstable (hence Hilbert unstable) if and only if $c>0$.

On the other hand, Xiao's method has been applied to this setting (regardless to the Maroni invariant) by Konno in [16], and leads to the bound (1.1).

This seems to suggest that the two methods of Cornalba-Harris and of Xiao, while being surprisingly similar in the case of fibred surfaces ( $c f$. [4]), become substantially different when applied to fibrations whose total space has dimension $\geq 3$.

Remark 3.6. As it is well known, gonality provides a stratification of the moduli space of smooth curves $\mathcal{M}_{g}$. Indeed, the loci

$$
\mathcal{D}_{k}:=\overline{\left\{[C] \in \mathcal{M}_{g} \text { such that } C \text { has a } g_{k}^{1}\right\}} \subseteq \mathcal{M}_{g}
$$

are closed subsets of $\mathcal{M}_{g}$ of decreasing codimension as $k$ goes from 2 to $[(g+3) / 2]$. The curves with maximal gonality $[(g+3) / 2]$ form an open set.

It has been proved ([1,9] in the semistable case) that if $f: S \longrightarrow B$ is a fibred surface of odd genus and such that the general fibres have maximal gonality, then $s(f) \geq 6(g-1) /(g+1)$. Moreover, the slope inequality $s(f) \geq 4(g-1) / g$, that holds for any fibred surface, is an equality only for some hyperelliptic fibrations. It seems therefore natural to conjecture an increasing bound for the slope of fibred surfaces depending on the gonality of the general fibres. A natural guess would be that the slope of non-hyperelliptic fibred surfaces should satisfy at least the bounds for trigonal fibrations (1.1). This is however false, as observed for instance in [2,25]: the easiest counterexamples are provided by bielliptic surfaces of arbitrarily large genus, with slope 4.

The right question to ask when looking for a bound increasing with gonality seems to be that the general fibres are "general" in the $k$-gonal locus: see [25, 
Conjecture 13.3]. From this point of view, the bound (1.3) could be the first step of the desired sequence.

It is worth mentioning that Konno proves the same bound (1.3) in [15, Corollary 4.4] under the assumptions that the fibration is non-hyperelliptic, and that $f_{*} \omega_{f}$ is a semistable vector bundle. The assumption of semistability for $f_{*} \omega_{f}$ is difficult to interpret; it would be very interesting to understand whether it is connected with some kind of "genericity" of the general fibre.

\section{References}

[1] T. AshiKaga and K. Konno, Global and local properties of pencils of algebraic curves, In: "Algebraic Geometry 2000", Azumino (Hotaka), Adv. Stud. Pure Math., Vol. 36, Math. Soc. Japan, Tokyo, 2002, 1-49.

[2] M. A. BARJA, "On the Slope and Geography of Fibred Surfaces and Threefolds", Ph. D. Thesis, Univesity of Barcelona, 1998.

[3] M. A. BARJA, On the slope of fibred threefolds, Internat. J. Math. 11 (2000), 461-491.

[4] M. A. BARJA and L. STOPPINO, Linear stability of projected canonical curves with applications to the slope of fibred surfaces, J. Math. Soc. Japan 60 (2008), 171-192.

[5] M. A. BARJA and F. ZuCCONI, On the slope of fibred surfaces, Nagoya Math. J. 164 (2001), 103-131.

[6] M. A. BARJA, Numerical bounds of canonical varieties, Osaka J. Math. (3) 37 (2000), 701-718.

[7] M. CORnAlba and J. HARRIS, Divisor classes associated to families of stable varieties, with applications to the moduli space of curves. Ann. Sci. École Norm. Sup. (4) 21 (1988), 455-475.

[8] W. Fulton, "Intersection Theory", second edition, Springer-Verlag, 1998.

[9] J. HARRIS and D. MUMFORD, On the Kodaira dimension of the moduli space of curves. With an appendix by William Fulton, Invent. Math. (1) 67 (1982), 23-88.

[10] R. Hartshorne, "Algebraic Geometry", GTM, Vol. 52, Springer-Verlag, New YorkHeidelberg, 1977.

[11] E. HoRIKAWA, On deformations of quintic surfaces, Invent. Math. 31 (1975), 43-85.

[12] Y. Kawamata, K. Matsuda and K. Matsuki, Introduction to the minimal model problem. In: "Algebraic Geometry", Sendai, 1985, Adv. Stud. Pure Math. 10, North Holland, Amsterdam, (1987), 283-360.

[13] G. R. KempF, Instability in invariant theory, Ann. of Math. 108 (1978), 299-316.

[14] H. KIM and Y. LEE, Log canonical thresholds of semistable plane curves, Math. Proc. Cambridge Philos. Soc. (2) 137 (2004), 273-280.

[15] K. Konno, Clifford index and the slope of fibered surfaces, J. Algebraic Geom. (2) 8 (1999), 207-220.

[16] K. Konno, A lower bound of the slope of trigonal fibrations, Internat. J. Math. (1) 7 (1996), 19-27.

[17] Y. LEE, Chow stability criterion in terms of log canonical threshold, J. Korean Math. Soc. (2) 45 (2008), 467-477.

[18] A. Maroni, Le serie lineari speciali sulle curve trigonali, Ann. Mat. Pura Appl. (4) 25 (1946), 341-354.

[19] G. MARTEns and F. O. Schreyer, Line bundles and syzygies of trigonal curves, Abh. Math. Sem. Univ. Hamburg 56 (1986), 169-189.

[20] I. Morrison, Projective stability of ruled surfaces, Invent. Math. (3) 56 (1980), 269-304.

[21] D. Mumford, Stability of projective varieties, L'Enseignement Math. (2) 23 (1977), 39-110. 
[22] K. OHNo, Some inequalities for minimal fibrations of surfaces of general type over curves, J. Math. Soc. Japan (4) 44 (1992), 643-666.

[23] M. REID, Chapters on algebraic surfaces, In: "Complex Algebraic Geometry" (Park City, UT, 1993), Amer. Math. Soc., Providence, RI, Vol. 3, 1997, 3-159.

[24] B. SAInt-Donat, On Petri's analysis of the linear system of quadrics through a canonical curve, Math. Ann. 206 (1973), 157-175.

[25] Z. E. Stankova-Frenkel, Moduli of trigonal curves, J. Algebraic Geom. (4) 9 (2000), $607-662$.

[26] L. Stoppino, Slope inequalities for fibered surfaces via GIT, Osaka Math. J. 45 (2008), 1027-1041.

[27] S. TAN, On the invariants of base changes of pencils of curves I, Manuscripta Math. 84 (1994), 225-244.

[28] S. TAN, On the invariants of base changes of pencils of curves II, Math. Z. 222 (1996), 655-676.

[29] S. TAN, On the slopes of the moduli spaces of curves, Internat. J. Math. 9 (1998), 119-127.

[30] G. Tian, The k-energy on hypersurfaces and stability, Comm. Anal. Geom. (2) 2 (1994), 239-265.

[31] E. VIEHWEG, “Quasi-projective Moduli for Polarized Manifolds”, Springer-Verlag, Ergebnisse der Mathematik und ihrer Grenzgebiete, Vol. 30, 1995.

[32] G. XIAO, Fibred algebraic surfaces with low slope, Math. Ann. 276 (1987), 449-466.

Departament de Matemàtica Aplicada I Universitat Politècnica de Catalunya ETSEIB Avda. Diagonal 08028 Barcelona, Spain

Miguel.Angel.Barja@upc.edu

Dipartimento di Fisica e Matematica Università dell'Insubria - Como Via Valleggio, 11 22100 Como, Italia lidia.stoppino@uninsubria.it 